\title{
Malondialdehyde-Modified LDL IgG Antibody Levels and Indices of Cardiac Function in Valvular Heart and Coronary Artery Disease Patients
}

\author{
Amir Ali Rahsepar ${ }^{a} \quad$ Asadollah Mirzaee $^{b, c}$ Fatemeh Moodi $^{b} \quad$ Mohsen Moohebati $^{b}$ \\ Shima Tavallaie ${ }^{a}$ Fatemeh Khorashadizadeh $^{\mathrm{e}}$ Behrooz Mottahedi $^{\mathrm{b}}$ Jamil Esfehanizadeh $^{\mathrm{b}}$ \\ Ali Azari ${ }^{b}$ Roshanak Khojasteh ${ }^{a}$ Somayeh Mousavi ${ }^{b}$ Roghayeh Paydar ${ }^{b}$ Maral Amini $^{\mathrm{b}}$ \\ Majid Ghayour-Mobarhan ${ }^{b}$ Gordon A. Ferns ${ }^{f}$ Amirhossein Sahebkar ${ }^{d, g}$

\begin{abstract}
${ }^{a}$ Biochemistry of Nutrition Research Center, ${ }^{b}$ Cardiovascular Research Center, School of Medicine, ${ }^{\mathrm{c}}$ Department of Cardiovascular Surgery, Ghaem Hospital, and ${ }^{\mathrm{d} B i o t e c h n o l o g y}$ Research Center, Mashhad University of Medical Sciences, Mashhad, and e Department of Epidemiology and Biostatistics, School of Public Health, Tehran University of Medical Sciences, Tehran, Iran; ${ }^{f}$ Brighton and Sussex Medical School, Division of Medical Education, Falmer, UK; ${ }^{9}$ Metabolic Research Centre, Royal Perth Hospital, School of Medicine and Pharmacology, University of Western Australia, Perth, W.A., Australia
\end{abstract}

\section{Key Words}

Anti-malondialdehyde-modified low-density lipoprotein . Valvular heart disease - Coronary artery bypass grafting · Cardiac function

\begin{abstract}
Objective: To compare the changes in anti-malondialdehyde-modified low-density lipoprotein (MDA-LDL) IgG levels among patients undergoing off-pump and on-pump coronary artery bypass grafting (CABG) or valvuloplasty. Subjects and Methods: A total of 38, 39 and 34 patients who underwent off-pump CABG, on-pump CABG and valvuloplasty, respectively, were enrolled in this study. Serum antiMDA-LDL IgG values were measured $24 \mathrm{~h}$ before and after the operative procedures and at discharge. Echocardiography was also done before surgery and before discharge. The results were compared with values from 50 healthy controls. Results: In all patients, a reduction in antibody titers was observed post-operatively. However, the decrease was signifi-
\end{abstract}

\section{KARGER 125\%}

E-Mail karger@karger.com www.karger.com/mpp
(C) 2015 S. Karger AG, Basel 1011-7571/15/0245-0424\$39.50/0

This is an Open Access article licensed under the terms of the Creative Commons Attribution-NonCommercial 3.0 Unported license (CC BY-NC) (www.karger.com/OA-license), applicable to the online version of the article only. Distribution permitted for non-commercial purposes only. cant only in the off-pump CABG - before surgery: 42.33 (25.83-58.51), after surgery: 30.86 (16.36-51.33) and at discharge: $10.96(6.82-23.57 ; p=0.027)$. There was a significant positive association between anti-MDA-LDL lgG levels and ejection fraction $(r=0.248, p=0.036)$ and a negative association with $E / E^{\prime}$, a marker of pulmonary capillary wedge pressure, in the coronary patients $(r=-0.345, p=0.012)$, but no significant associations were found in patients with valvular heart disease. Conclusions: Serum anti-MDA-LDL IgG levels were associated with cardiac function indices in coronary patients undergoing $C A B G$.

๑) 2015 S. Karger AG, Basel

\section{Introduction}

Myocardial revascularization can be undertaken using minimally invasive surgical techniques or by open surgical treatment, known as coronary artery bypass grafting $(\mathrm{CABG})$. The latter can be performed with or 
without the use of cardiopulmonary bypass (CPB) defined as 'on-pump' and 'off-pump' CABG, respectively. Degenerative and rheumatic valvular heart disease (VHD) has several risk factors in common with coronary artery disease (CAD); these include genetic, inflammatory and autoimmune determinants, infection and oxidative stress $[1,2]$.

Both heart valvular replacement and on-pump CABG require $\mathrm{CPB}$. Valvular replacement and coronary revascularization with the use of $\mathrm{CPB}$ comprise a comparable intraoperative procedure. One of the complications that may occur following $\mathrm{CPB}$ is the ischemia-reperfusion syndrome [3]. During this condition, functional, structural and metabolic changes occur, one of which is an increase in the production of free radicals [3], which may lead to cell necrosis or apoptosis in cardiac tissue. The use of off-pump CABG has been suggested to reduce ischemia-reperfusion injury in patients with acute coronary syndrome, thereby improving the outcomes following surgery [4]. It has been suggested that the avoidance of CPB may prevent subsequent ischemia-reperfusion injury and hence reduce postoperative systemic complications $[5,6]$.

Several potential autoantigens have been identified that may be involved in the pathogenesis of atherosclerosis. These include oxidatively modified low-density lipoprotein (LDL), for example by malondialdehyde (MDA), heat shock proteins and $\beta_{2}$-glycoprotein-I $[7,8]$. There are several epitopes of MDA-LDL that induce an autoimmune response in man. In vitro modified human LDL, e.g. copper-oxidized LDL (copper-ox-LDL) and MDAmodified LDL have been shown to elicit an immune response in animals and humans, resulting in the formation of autoantibodies [9-11].

Hence, the objective of this current study was to assess the changes in serum anti-MDA-LDL IgG autoantibodies in relation to indices of cardiac function in patients undergoing $C A B G$ and heart valvular replacement and to compare the changes in anti-MDA-LDL IgG autoantibodies amongst patients who were treated by different operative procedures.

\section{Subjects and Methods}

\section{Study Population}

The study subjects $(\mathrm{n}=111)$ were selected from individuals who were candidates for open heart surgery and had proven CAD or VHD. All the subjects had previously undergone coronary angiography. The indication for coronary revascularization and the type of surgery (off- or on-pump CABG) was determined on the basis of clinical judgment by the attending cardiac surgeon. In total, 38 patients were recruited to the off-pump CABG group, 39 patients to the on-pump CABG group and 34 patients to the valvuloplasty group. The patients with VHD had one or two diseased valves. Percutaneous coronary angiography was indicated for all patients with VHD in order to rule out the presence of CAD. The angiography showed that all the patients with VHD were free from significant $\mathrm{CAD}$. The exclusion criteria were pregnancy, consumption of oral contraceptives, a positive result for viral markers of hepatitis and anti-HIV antibody, a prior history of coronary angioplasty or $\mathrm{CABG}$, chronic pulmonary obstructive disease, overt clinical features of infection or chronic inflammatory disease, hormone replacement therapy, patients with acute coronary syndrome within the previous 3 months, renal, hepatic or malignant diseases, and cases admitted as emergencies.

A total of 50 healthy volunteers were recruited as a normal control group. The control group comprised subjects who had never experienced any symptom or had any signs of CVD. These subjects had no other known obvious major disease. The data for the control group were collected from an ongoing prospective study. Information on smoking and drug treatment was obtained using a questionnaire. Demographic and intraoperative data were recorded, including aortic cross-clamp time, CPB time, duration of surgery, and number of grafts. Echocardiography was performed for all patients. The study protocol was approved by the Ethics Committee of the Mashhad University of Medical Sciences, and written informed consent was obtained from each participant.

The following conventional cardiovascular risk factors were defined: dyslipidemia [total cholesterol $\geq 200 \mathrm{mg} / \mathrm{dl}$ and/or LDLcholesterol (LDL-C) $\geq 130 \mathrm{mg} / \mathrm{dl}$ and/or use of cholesterol-lowering drugs], diabetes (fasting blood glucose $\geq 126 \mathrm{mg} / \mathrm{dl}$ and/or use of antihyperglycemic treatment) and arterial hypertension (systolic blood pressure $>140 \mathrm{~mm} \mathrm{Hg}$ and/or diastolic blood pressure $>90 \mathrm{~mm} \mathrm{Hg}$ and/or use of antihypertensive drugs).

\section{Anthropometric and Other Measurements}

For all patients, anthropometric parameters, including weight and height, were measured preoperatively, and the body mass index (BMI) was calculated (in kilograms/square meters). Blood pressure was measured twice while the patients were seated and rested, using a standard mercury sphygmomanometer.

\section{Blood Sampling and Routine Biochemical Analysis}

The first blood sample was taken from each patient for analysis after $12 \mathrm{~h}$ of fasting in the morning on the day of surgery. The second sample was taken within the first $24 \mathrm{~h}$ after surgery in the ICU from a peripheral vein. The third blood sample was taken on the day of discharge. Following venipuncture, blood samples were collected into Vacutainer ${ }^{\circledR}$ tubes and centrifuged at 5,000 $\mathrm{g}$ for $15 \mathrm{~min}$ at $4^{\circ} \mathrm{C}$. After separation, aliquots of serum were frozen at $-80^{\circ} \mathrm{C}$ until analysis. A full-fasted lipid profile comprising total cholesterol, triglycerides, high-density lipoprotein-cholesterol (HDL-C) and LDL-C was determined for each subject. Serum lipid and fasting blood glucose concentrations were measured enzymatically with the use of commercial kits using a BT-3000 autoanalyzer (Biotechnica, Rome, Italy).

\section{MDA-LDL IgG Measurements}

Anti-MDA-LDL IgG levels were measured using an antiMDA-LDL ELISA kit (Immundiagnostik AG, Bensheim, Germa- 
ny) - a sandwich ELISA for the direct measurement of MDA-LDL IgG antibodies in human plasma and serum. Standards, controls and samples containing human anti-MDA-LDL antibodies were added to the wells of a microplate coated with MDA-LDL. Following the first incubation period $(2 \mathrm{~h}$ at room temperature on a horizontal mixer), unbound components were washed away, and a peroxidase-labeled conjugate was added to each microtiter well. Tetramethylbenzidine was then added as a peroxidase substrate. Finally, an acidic stop solution was added to terminate the colorimetric reaction. The intensity of the yellow color was measured and was directly proportional to the MDA-LDL IgG concentration in the sample. Anti-MDA-LDL IgG concentration in the samples was determined directly from the standard curve of absorbance unit (optical density at $450 \mathrm{~nm}$ ) versus concentration.

\section{Cardiopulmonary Bypass}

The CPB was instituted by cannulation of the distal ascending aorta and insertion of a single 2-stage cannula into the right atrium. A membrane oxygenator (Didico; Surin Group, Arvada, Colo., USA) was used. Intravenous heparin $(300 \mathrm{IU} / \mathrm{kg}$ ) was administered immediately before cannulation for CPB (Stockert SIII and SV; Medtronic, USA), and additional doses were given to maintain an activated clotting time of $480 \mathrm{~s}$ or more. Nonpulsatile flow rates of 2.2-2.4 liters $/ \mathrm{min} / \mathrm{m}^{2}$ and temperatures between 28 and $30^{\circ} \mathrm{C}$ were used. The mean arterial pressure was maintained between 50 and $60 \mathrm{~mm} \mathrm{Hg}$, with the administration of sodium nitroprusside or norphenylephrine as required, and the hematocrit was kept higher than $27 \%$ by adding concentrated red blood cells if necessary. A 14Fr retrograde coronary sinus perfusion catheter (Callmed, USA) was inserted by palpation of the coronary sinus for blood collection and retrieving coronary sinus blood samples. The basic cardioplegic solution used in both groups was prepared by mixing $500 \mathrm{ml}$ of whole blood withdrawn directly from the pump oxygenator to a reservoir to which $\mathrm{KCl}(10 \mathrm{mmol})$, lidocaine hydrochloride $(60 \mathrm{mg})$ and magnesium sulfate $(8 \mathrm{mmol})$ were added. After cross-clamping of the ascending aorta, cardioplegia was induced in all patients by antegrade infusion of $1,000 \mathrm{ml}$ of the cold cardioplegic solution obtained by use of a roller pump. Additional doses of $600 \mathrm{ml}$ were infused after each distal anastomosis, or after $20 \mathrm{~min}$ of ischemia, and immediately before releasing the aortic cross-clamp.

During off-pump surgery, all operations were performed through a median sternotomy. Intravenous heparin $(150 \mathrm{IU} / \mathrm{kg})$ was administered. All anastomoses were sutured by hand. The proximal anastomosis was performed using a site clamp on the aorta. At the end of the surgical procedure, protamine sulfate was administered to reverse the heparin effect for both on-pump and off-pump patients.

\section{Cardiac and Vascular Echocardiography}

Resting echocardiographic examination was done for all of the patients before surgery and on the day of discharge using VIVID 3 (GE Vingmed Ultrasound, USA). Left ventricular ejection fraction (EF), left ventricular end systolic and diastolic diameters and volumes, early (E) and late (A) mitral forward Doppler flow, early (E') and late (A') diastolic mitral annulus pulsed-wave tissue Doppler, and end systolic and diastolic volume were measured. The E/E' ratio indicated the pulmonary capillary wedge pressure. Diastolic functions, as described previously [12], were categorized as normal, stage I (impaired), stage II (pseudonormal), and stage III (restrictive).

\section{Statistical Analysis}

All statistical analyses were performed using the SAS for Windows $^{\mathrm{TM}}$, version 9.2 software package (Cary, N.C., USA). Data were expressed as means $\pm \mathrm{SD}$ (for parameters with a normal distribution) or medians and interquartile ranges (in the case of nonnormally distributed data). For parameters with a normal distribution group comparisons were performed using the Student $t$ test (between 2 groups) and ANOVA (for $\geq 3$ groups). For parameters with a nonnormal distribution and unequal variances, and for ordinal variables, Mann-Whitney (between 2 groups) and KruskalWallis (for $\geq 3$ groups) tests were used. A two-sided $p$ value of $<0.05$ was considered to be statistically significant. Bivariate correlations between different parameters and anti-MDA-LDL IgG levels were performed using Spearman's rank correlation (for nonnormally distributed data) or Pearson's correlation coefficients (for normally distributed data). Significant associations were adjusted for confounders (including age, gender, smoking, and BMI) using generalized linear models. In order to compare anti-MDALDL IgG levels in the 3 samples of the patients among the on- and off-pump CABG and VHD groups, a repeated measure test was used. The Wilcoxon signed-rank test was used to compare withingroup changes. Due to the presence of some missing data, we used Proc Mixed in SAS based on the likelihood that with a few missing observations we may lose a great deal of data and degrees of freedom.

\section{Results}

\section{Demographic Data}

The demographic and biochemical characteristics of the patients and controls are summarized in table 1 . The first and second echocardiographic findings of patients are summarized in table 2 . The interassay coefficient of variation for the measurement of serum MDA-LDL IgG was $1.9 \%$.

The baseline levels of serum anti-MDA-LDL IgG were not statistically different between patients either with CAD [on-pump group: 34.46 (19.60-51.74), off-pump group: 42.33 (25.83-58.51)] or VHD [44.46 (26.0258.56)] and controls [40.96 (29.94-56.91; p > 0.05)]. Overall, in the 2 groups of CABG patients, a reduction in antibody titers was observed as follows: on-pump group - before surgery: 34.46 (19.60-51.74), after surgery: 30.55 (16.63-45.56) and at discharge: 24.43 (19.1153.85); off-pump group - before surgery: 42.33 (25.8358.51), after surgery: $30.86(16.36-51.33)$ and at discharge: 10.96 (6.82-23.57). A reduction in antibody titers was also observed for VHD - before surgery: 44.46 (26.02-58.56), after surgery: 37.57 (19.51-57.71) and at discharge: 35.58 (28.90-41.57; fig. 1).

The bivariate analysis did not reveal any significant association between age, anthropometric factors and biochemical measurements with antibody levels in the pa- 


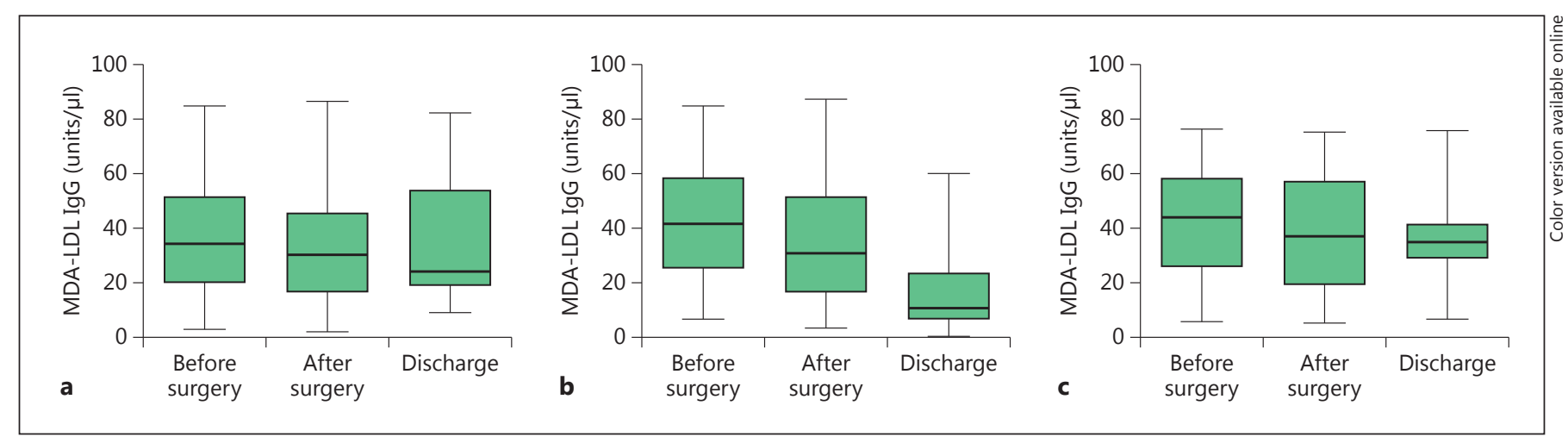

Fig. 1. Comparison of anti-MDA-LDL IgG levels before surgery, after surgery and at discharge in the on-pump (a), off-pump (b) and VHD (c) study groups. The box and whisker plots show range and quartiles. The boxes extend from the 25 th to the 75 th percentile, with a line at the median. The whiskers show the highest and the lowest values.

Table 1. Comparison of clinical and biochemical characteristics of patients and controls

\begin{tabular}{lccc}
\hline & CAD patients $(\mathrm{n}=77)$ & VHD patients $(\mathrm{n}=34)$ & Controls $(\mathrm{n}=50)$ \\
\hline Female & $30(38.96)$ & $17(50)$ & $22(44)$ \\
Smokers & $22(28.57)$ & $8(24)$ & $6(12)^{\mathrm{a}}$ \\
Diabetics & $29(37.66)$ & $0(0)$ & $1(2)^{\mathrm{a}}$ \\
Dyslipidemia & $39(50.65)$ & $3(9)$ & $4(8)^{\mathrm{a}}$ \\
Hypertension & $50(64.93)$ & $6(18)$ & $7(14)^{\mathrm{a}}$ \\
Age, years & $60.36 \pm 9.26$ & $43.50 \pm 13.63$ & $55.54 \pm 7.87^{\mathrm{a}, \mathrm{b}}$ \\
BMI & $27.25(24.15-29.75)$ & $23.08(19.78-26.12)$ & $26.75(24.52-30.92)^{\mathrm{b}}$ \\
Waist circumference, cm & $96.5(89-103)$ & $90(77-96)$ & $96(90-107.75)^{\mathrm{b}}$ \\
Hip circumference, cm & $104.5(98-110)$ & $98(87-103)$ & $104(97-108)^{\mathrm{b}}$ \\
Fasting blood glucose, mg/dl & $110.5(95.75-138.75)$ & $95(84-112)$ & $88(78-97)^{\mathrm{a}, \mathrm{b}}$ \\
Triglycerides, mg/dl & $143(86-197)$ & $101(84-124)$ & $119(94-171.25)^{\mathrm{b}}$ \\
HDL-C, mg/dl & $42(34-47)$ & $43(35.5-51.5)$ & $46(42-51.22)^{\mathrm{a}}$ \\
LDL-C, mg/dl & $97.3(73.45-120.25)$ & $91.2(75.35-115.27)$ & $97.25(85-116.57)$ \\
DBP, mm Hg & $80(70-80)$ & $70(66.25-84.25)$ & $70(70-85)^{\mathrm{b}}$ \\
SBP, mm Hg & $127.5(120-130)$ & $115(110-120)$ & $120(115-130)^{\mathrm{b}}$ \\
\hline
\end{tabular}

Values are expressed as $\mathrm{n}(\%)$, means $\pm \mathrm{SD}$ or medians (with interquartile ranges), as appropriate. $\mathrm{DBP}=$ Diastolic blood pressure; SBP $=$ systolic blood pressure. ${ }^{a} \mathrm{p}<0.05$ : mean significant difference between CAD patients and controls; ${ }^{\mathrm{b}} \mathrm{p}<0.05$ : mean significant difference between VHD patients and controls ( $\chi^{2}$ test and MannWhitney test were used).

tients with CAD and VHD. There was a significant positive association between baseline anti-MDA-LDL IgG levels, with a median of 38.22 (interquartile range: $22.94-$ 53.98), and $\mathrm{EF}$ (mean \pm SD: $51.37 \pm 10.47 ; \mathrm{r}=0.248, \mathrm{p}=$ 0.036 ) and a negative association with E/E' (mean \pm SD: $10.89 \pm 4.63 ; \mathrm{r}=-0.345, \mathrm{p}=0.012)$ in the coronary patients but not in VHD patients (fig. 2). These correlations remained significant after adjustment for confounders that included age, gender, smoking, and BMI ( $\mathrm{p}=0.046$ with $\mathrm{EF}$, and $\mathrm{p}=0.041$ with $\left.\mathrm{E} / \mathrm{E}^{\prime}\right)$.

Anti-MDA-LDL IgG and Cardiac Function

\section{Discussion}

A novel finding of the present study was that serum anti-MDA-LDL IgG levels in CAD patients were positively associated with $\mathrm{EF}$ and negatively associated with E/E' (a marker of capillary wedge pressure in the coronary patients). The VHD patients had higher E/E' values compared with CAD patients because of their valvular heart pathology. In the VHD group, no significant association was observed between anti-MDA-LDL IgG levels and 
Table 2. Echocardiographic findings and pump and aortic clamp time of patients

\begin{tabular}{|c|c|c|c|}
\hline & On-pump $(\mathrm{n}=39)$ & Off-pump $(\mathrm{n}=38)$ & VHD $(\mathrm{n}=34)$ \\
\hline Pre-EF, \% & $50(41-56)$ & $55(50-60)$ & $55(50-60)$ \\
\hline Post-EF, \% & $45(35-57)$ & $50(45-55)$ & $51.11 \pm 9.44$ \\
\hline Pre-E, cm/s & $60(50-77.5)$ & $70(50-83)$ & $115(79-210)^{\mathrm{a}}$ \\
\hline Post-E, $\mathrm{cm} / \mathrm{s}$ & $70(59-99)$ & $70(58-85)$ & $107(77-145)$ \\
\hline Pre-E', cm/s & $6(4-7)$ & $6.5(6-8)$ & $7(5-10)$ \\
\hline Post-E,' cm/s & $5(5-7)$ & $6.5(5-7)$ & $7.8(5-9.2)$ \\
\hline Pre-A, $\mathrm{cm} / \mathrm{s}$ & $92(74-104)$ & $79.5(69.25-90)$ & $90(56-149)$ \\
\hline Post-A, cm/s & $80(70.5-87.5)$ & $70(60.5-80)$ & $72(58-93)$ \\
\hline Pre-A', cm/s & $9(6.5-10)$ & $10(8-11)$ & $7(6-10)^{a}$ \\
\hline Post-A', cm/s & $7(5-8.1)$ & $8(7-10)$ & $5(5-6)$ \\
\hline Pre-E/E' & $10.16(7.81-12.43)$ & $10(7.96-11.95)$ & $15.8(9.55-35.83)^{\mathrm{a}}$ \\
\hline Post-E/E’ & $12.17(10.57-19.67)$ & $10.47(8.36-13.81)$ & $15.29(10.36-21.13)$ \\
\hline Pre-LVDD, mm & $49.8(45.5-59)$ & $50(47-55.1)$ & $48(40-53)$ \\
\hline Post-LVDD, mm & $51(47-58)$ & $47(40-58.75)$ & $44.85(41-51.75)$ \\
\hline Pre-LVSD, mm & $36(29-43)$ & $36(30.8-39.25)$ & $34.4(28.25-42)$ \\
\hline Post-LVSD, mm & $38.5(32.22-44.75)$ & $31(28-40.75)$ & $30.75(27.77-39.23)$ \\
\hline Pre-EDV, ml & $79(59-93.5)$ & $84(60-100)$ & $79.5(50.75-95.43)$ \\
\hline Post-EDV, ml & $87.5(60-123)$ & $77(59.25-91.75)$ & $82(64.5-109)$ \\
\hline Pre-ESV, ml & $30(25.25-39.75)$ & $34(23-44)$ & $35(20-58)$ \\
\hline Post-ESV, ml & $38(25.25-57.75)$ & $35.5(23-45.75)$ & $42(27.5-50.65)$ \\
\hline \multicolumn{4}{|l|}{ Prediastolic function } \\
\hline Normal & $2(7.4)$ & $7(25.9)$ & $7(32)$ \\
\hline Grade I & $22(81.5)$ & $16(59.3)$ & $7(32)$ \\
\hline Grade II & $2(7.4)$ & $3(11.1)$ & $6(27)$ \\
\hline Grade III & $1(3.7)$ & $1(3.7)$ & $2(9)$ \\
\hline \multicolumn{4}{|l|}{ Postdiastolic function } \\
\hline Normal & $1(5.9)$ & $4(14.3)$ & $6(37.50)$ \\
\hline Grade I & $11(64.7)$ & $15(53.6)$ & $7(43.75)$ \\
\hline Grade II & $4(23.5)$ & $9(32.1)$ & $3(18.75)$ \\
\hline Grade III & $1(5.9)$ & $0(0)$ & $0(0)$ \\
\hline Aorta clamp time, min & $50(45-57)$ & - & $52(33.5-64)$ \\
\hline Pump time, $\min$ & $88(72.25-118.25)$ & - & $74(57-114)$ \\
\hline
\end{tabular}

Values are expressed as n (\%) or medians (with interquartile ranges), as appropriate. ESV = End systolic volume; $\mathrm{EDV}=$ end diastolic volume; LVDD = left ventricular diastolic diameter; LVSD = left ventricular systolic diameter. Note: E and E' stand for peak early transmitral flow velocity and peak early diastolic myocardial velocity, respectively. E/E' means pulmonary capillary wedge pressure. The Kruskal-Wallis test was used to compare the results among the 3 groups. Preechocardiographic findings were compared among the 3 groups. ${ }^{a} \mathrm{p}<0.05$ : pre-E, pre-E/E' and pre-A' were significantly different between the 3 groups of patients.

measures of cardiac function (EF and E/E'). There have been several reports investigating the association between cardiac function and oxidative stress. It has been previously reported that increased levels of serum markers of oxidative stress correlate inversely with left ventricular EF in patients with ischemic and nonischemic dilated cardiomyopathy $[13,14]$. Belch et al. [15] have also reported a negative correlation between left ventricular EF and oxidative stress.
In this study, the nonstatistically significant difference in anti-MDA-LDL IgG levels between patients with VHD and CAD compared with healthy controls is consistent with previous studies [16-20]. McDowell et al. [18] did not find a significant difference in serum IgG titers to oxLDL between patients with CAD and healthy subjects. They also reported no correlation between anti-ox-LDL antibodies and coronary atherosclerosis [18]. In another study [19], serum anti-ox-LDL concentration was determined in patients with severe and minor atherosclerosis, 

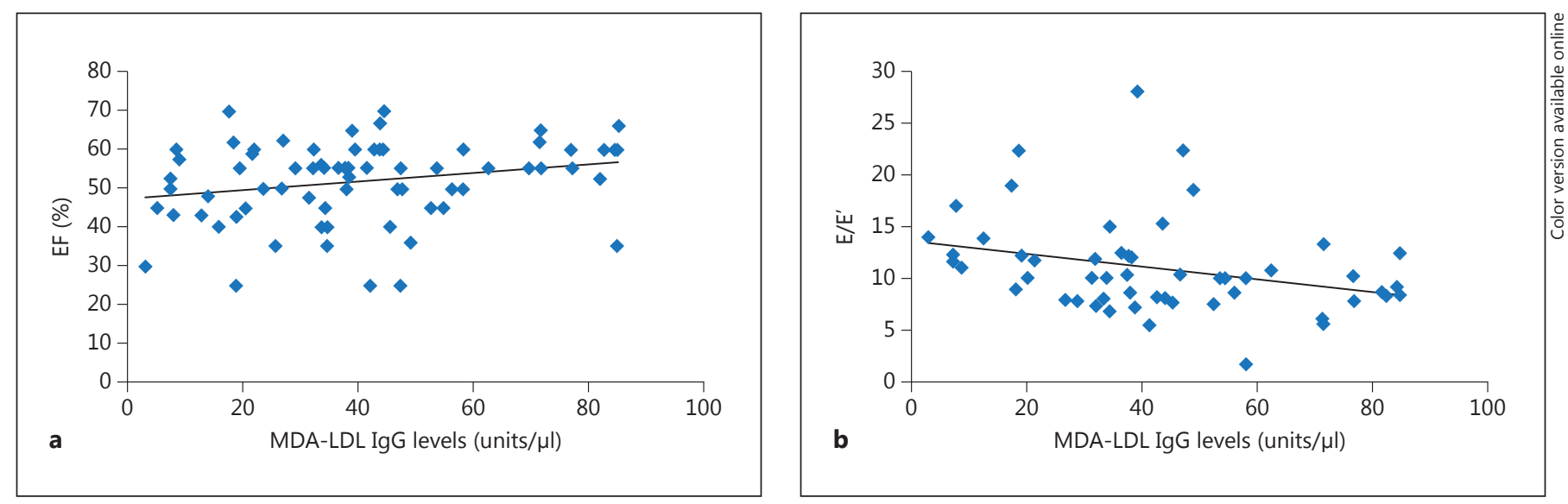

Fig. 2. Significant correlations between baseline serum anti-MDA-LDL IgG levels and echocardiographic measures $\mathrm{EF}(\mathbf{a})$ and $\mathrm{E} / \mathrm{E}^{\prime}(\mathbf{b})$.

as well as in healthy subjects. It was reported that anti-oxLDL concentration was not significantly different between the above-mentioned 3 groups, and there was no association between anti-ox-LDL concentration and severity of aortic stenosis [19]. Similar to these findings, a recent study also showed that serum anti-ox-LDL titers were comparable between patients with CAD and healthy subjects, and the antibody titers were not associated with the severity of CAD according to the number of narrowed vessels [20].

In spite of a significant reduction in plasma MDALDL IgG levels in the off-pump group, between-group comparison of changes did not suggest a significant difference between the on- and off-pump groups. This finding is contradictory to the results of a previous study, in which an increase in LDL oxidation was found $48 \mathrm{~h}$ after $\mathrm{CPB}$ compared with the presurgery state [20]. Moreover, Lazzarino et al. [21] reported that after intermittent reperfusion during the aortic cross-clamp, a 10-fold increase in net myocardial MDA production occurred, suggesting that myocardial lipid peroxidation, estimated through MDA measurement, is common during intermittent ischemia-reperfusion in patients undergoing CABG [21]. In the same study, an increase in MDA concentration was found in the coronary sinus blood during the short intermittent reperfusion periods as well as $2 \mathrm{~min}$ after the aortic cross-clamp [21]. In contrast, Bical et al. [22] showed that preischemic myocardial levels of MDA were higher than levels at the end of the aortic crossclamp period or after $30 \mathrm{~min}$ of reperfusion. However, in another study, the MDA concentration was not changed

Anti-MDA-LDL IgG and Cardiac

Function after initiating CPB [23]. MDA levels have been reported to be significantly increased in patients undergoing $\mathrm{CPB}$ during on-pump CABG $[24,25]$. Tsimikas et al. [26] measured MDA-LDL IgG levels before percutaneous coronary intervention (PCI) and at serial time points after intervention. They showed that IgG autoantibodies to MDA-LDL are reduced immediately after PCI but return to normal by $6 \mathrm{~h}$ after intervention. This finding is consistent with the results observed in the present study in the on-pump CABG group, in which no significant difference was found between pre-CABG MDA-LDL IgG levels and values at $24 \mathrm{~h}$. However, the present study design is unable to clarify whether there was any acute reduction in MDA-LDL IgG levels after CABG as blood sampling and antibody assay were not performed at serial time points immediately after PCI. It is rational to consider an acute reduction in MDA-LDL IgG levels as a result of complexation of antibodies with MDA-LDL that is released from plaque compression and disruption due to $\mathrm{CABG}$ and postangioplasty ischemia/reperfusion. Nevertheless, rapid elevation of MDA-LDL IgG levels in $<6 \mathrm{~h}$ after PCI, as reported by Tsimikas et al. [26], needs to be verified by additional studies because the kinetics of antibody production by memory B cells is unlikely to allow interpretations based on increased MDA-LDL IgG production. It might be speculated that redistribution of antigen-antibody complexes from the hepatic tissue into plasma could be responsible for such a rapid increase in MDA-LDL IgG levels, but this hypothesis needs to be verified in future studies. In contrast with the on-pump and CABG groups, a decreasing trend in MDA-LDL IgG lev- 
els was observed in the off-pump group. This finding may be attributed to the CPB-free procedure of off-pump surgery (contrary to on-pump surgery and valvuloplasty) because CPB may induce postoperative systemic inflammatory and oxidative responses [27]. The clinical relevance of such a decrease in MDA-LDL IgG levels following offpump CABG is open to question owing to the case-control design and also the relatively small population size that was included in this study.

ox-LDL is removed by antioxidant mechanisms effectively and rapidly, and it has been previously reported that ox-LDL is not present at significant concentrations in the circulation [28]. In an animal study, it was shown that the content of ox-LDL in arterial tissue correlates with autoantibodies present in the blood against ox-LDL [29]. In the present study, MDA-LDL IgG levels fell in all groups postoperatively, and this reduction was significant in VHD but not coronary patients. It appears that oxidative stress associated with surgery may lead to increased MDA-LDL production and the formation of antibody-antigen complexes that are rapidly removed from the circulation via the reticuloendothelial system. The present results are similar to our previous study in which the antibody levels against heat shock protein-27, another possible risk factor for atherosclerosis, were found to be decreased $12 \mathrm{~h}$ after the onset of acute coronary syndrome [30], and it was proposed that this rapid reduction may have been attributed to the formation of antibodyantigen complexes following the necrosis of cardiac tissue [30].

The major limitation of the present study is lack of randomization. It was not possible to randomize patients to different surgeries, and this may have resulted in a selection bias owing to differences in disease severity and other potential confounding factors between the study groups. Another limitation is that oxidative burden as well as vascular injury following CABG or heart valve replacement may not be fully represented by serum levels of anti-MDA-LDL IgG levels. Hence, measurement of additional indicators of oxidative stress (e.g. MDA, isoprostanes and prooxidant-antioxidant balance is recommended to be performed in future studies aiming to verify the present results. Finally, anti-MDA-LDL IgG levels were determined in only 2 postsurgical samples in the present study. In order to obtain a clearer picture on the pattern of changes and kinetics of anti-MDA-LDL IgG levels, it would be ideal to evaluate the changes in serial samples obtained immediately after CABG.

\section{Conclusions}

In the present study, serum anti-MDA-LDL IgG levels in coronary patients were associated with indices of cardiac function in coronary patients. The clinical relevance of such an association needs to be verified in longitudinal studies (prospective cohorts) in order to clarify whether raised MDA-LDL IgG levels can significantly predict cardiovascular outcomes.

\section{Acknowledgments}

This work was supported by Mashhad University of Medical Sciences, Iran. The results presented in this work were taken from a thesis by Amir Ali Rahsepar at Mashhad University of Medical Sciences (ID No. 87065). We are also particularly grateful to the patients and their family members who volunteered to participate in this study.

\section{Disclosure Statement}

The authors have no conflicts of interest to declare.

\section{References}

1 O'Brien KD: Pathogenesis of calcific aortic valve disease: a disease process comes of age (and a good deal more). Arterioscler Thromb Vasc Biol 2006;26:1721-1728.

2 Davutoglu V, Celik A, Aksoy M: Contribution of selected serum inflammatory mediators to the progression of chronic rheumatic valve disease, subsequent valve calcification and NYHA functional class. J Heart Valve Dis 2005; 14:251-256
3 Vaage J, Valen G: Pathophysiology and mediators of ischemia-reperfusion injury with special reference to cardiac surgery. A review. Scand J Thorac Cardiovasc Surg Suppl 1993; 41:1-18.

4 Rastan AJ, Eckenstein JI, Hentschel B, et al: Emergency coronary artery bypass graft surgery for acute coronary syndrome: beating heart versus conventional cardioplegic cardiac arrest strategies. Circulation 2006; 114:I477-I485.
5 Cleveland JC Jr, Shroyer AL, Chen AY, et al: Off-pump coronary artery bypass grafting decreases risk-adjusted mortality and morbidity. Ann Thorac Surg 2001;72:1282-1288.

6 Sabik JF, Gillinov AM, Blackstone EH, et al: Does off-pump coronary surgery reduce morbidity and mortality? J Thorac Cardiovasc Surg 2002;124:698-707.

-7 Mandal K, Jahangiri M, Xu Q: Autoimmune mechanisms of atherosclerosis. Handb Exp Pharmacol 2005;170:723-743. 
8 Ghayour-Mobarhan M, Rahsepar AA, Tavallaie S, et al: The potential role of heat shock proteins in cardiovascular disease: evidence from in vitro and in vivo studies. Adv Clin Chem 2009;48:27-72.

-9 Virella G, Thorpe SR, Alderson NL, et al: Definition of the immunogenic forms of modified human LDL recognized by human autoantibodies and by rabbit hyperimmune antibodies. J Lipid Res 2004;45:1859-1867.

10 Su J, Georgiades A, Wu R, et al: Antibodies of IgM subclass to phosphorylcholine and oxidized LDL are protective factors for atherosclerosis in patients with hypertension. Atherosclerosis 2006;188:160-166.

11 Virella G, Carter RE, Saad A, et al: Distribution of IgM and IgG antibodies to oxidized LDL in immune complexes isolated from patients with type 1 diabetes and its relationship with nephropathy. Clin Immunol 2008; 127 : 394-400.

12 Garcia MJ, Thomas JD, Klein AL: New Doppler echocardiographic applications for the study of diastolic function. J Am Coll Cardiol 1998;32:865-875.

13 Harrison D, Griendling KK, Landmesser U, et al: Role of oxidative stress in atherosclerosis. Am J Cardiol 2003;91:7A-11A.

14 Vassalle C, Petrozzi L, Botto N, et al: Oxidative stress and its association with coronary artery disease and different atherogenic risk factors. J Intern Med 2004;256:308-315.

$\checkmark 15$ Belch JJ, Bridges AB, Scott N, et al: Oxygen free radicals and congestive heart failure. $\mathrm{Br}$ Heart J 1991;65:245-248.
6 Pandya DP: Oxidant injury in coronary heart disease. Part I. Compr Ther 2001;27:284-292.

17 Sayar N, Terzi S, Yilmaz HY, et al: Exerciseinduced increase in lipid peroxidation in patients with chronic heart failure: relation to exercise intolerance. Cardiology 2007;108: 307-313.

18 McDowell A, Young IS, Wisdom GB: Autoantibodies to malondialdehyde-modified low-density lipoprotein in patients with angiographically confirmed coronary artery disease. J Pharm Pharmacol 2002;54:1651-1657.

19 van de Vijver LP, Steyger R, van PG, et al: Autoantibodies against MDA-LDL in subjects with severe and minor atherosclerosis and healthy population controls. Atherosclerosis 1996; 122:245-253.

20 Jahangiri M, Kovacs IB, Ridler CD, et al: Coronary artery surgery is associated with different forms of atherogenic lipoprotein modifications. Ann Thorac Surg 1999;67:652-656.

21 Lazzarino G, Raatikainen P, Nuutinen M, et al: Myocardial release of malondialdehyde and purine compounds during coronary bypass surgery. Circulation 1994;90:291-297.

22 Bical O, Gerhardt MF, Paumier D, et al: Comparison of different types of cardioplegia and reperfusion on myocardial metabolism and free radical activity. Circulation 1991; 84:III375-III379.

23 Toivonen HJ, Ahotupa M: Free radical reaction products and antioxidant capacity in arterial plasma during coronary artery bypass grafting. J Thorac Cardiovasc Surg 1994;108: 140-147.

24 Koksal H, Rahman A, Burma O, et al: The effects of low dose $\mathrm{N}$-acetylcysteine (NAC) as an adjunct to cardioplegia in coronary artery bypass surgery. Anadolu Kardiyol Derg 2008; 8:437-443.
25 Rodrigues AJ, Evora PR, Bassetto S, et al: Blood cardioplegia with $\mathrm{N}$-acetylcysteine may reduce coronary endothelial activation and myocardial oxidative stress. Heart Surg Forum 2009;12:E44-E48.

26 Tsimikas S, Lau HK, Han KR, et al: Percutaneous coronary intervention results in acute increases in oxidized phospholipids and lipoprotein(a): short-term and long-term immunologic responses to oxidized low-density lipoprotein. Circulation 2004;109:3164-3170.

27 Cleveland JC Jr, Shroyer AL, Chen AY, et al: Off-pump coronary artery bypass grafting decreases risk-adjusted mortality and morbidity. Ann Thorac Surg 2001;72:1282-1288, discussion 1288-1289.

-28 van Berkel TJ, De Rijke YB, Kruijt JK: Different fate in vivo of oxidatively modified low density lipoprotein and acetylated low density lipoprotein in rats. Recognition by various scavenger receptors on Kupffer and endothelial liver cells. J Biol Chem 1991;266:22822289.

-29 Tsimikas S, Palinski W, Witztum JL: Circulating autoantibodies to oxidized LDL correlate with arterial accumulation and depletion of oxidized LDL in LDL receptor-deficient mice. Arterioscler Thromb Vasc Biol 2001;21:95100.

-30 Ghayour-Mobarhan M, Sahebkar A, Parizadeh SM, et al: Antibody titres to heat shock protein 27 are elevated in patients with acute coronary syndrome. Int J Exp Pathol 2008;89: 209-215. 